SPACE

\title{
More Science from Apollo
}

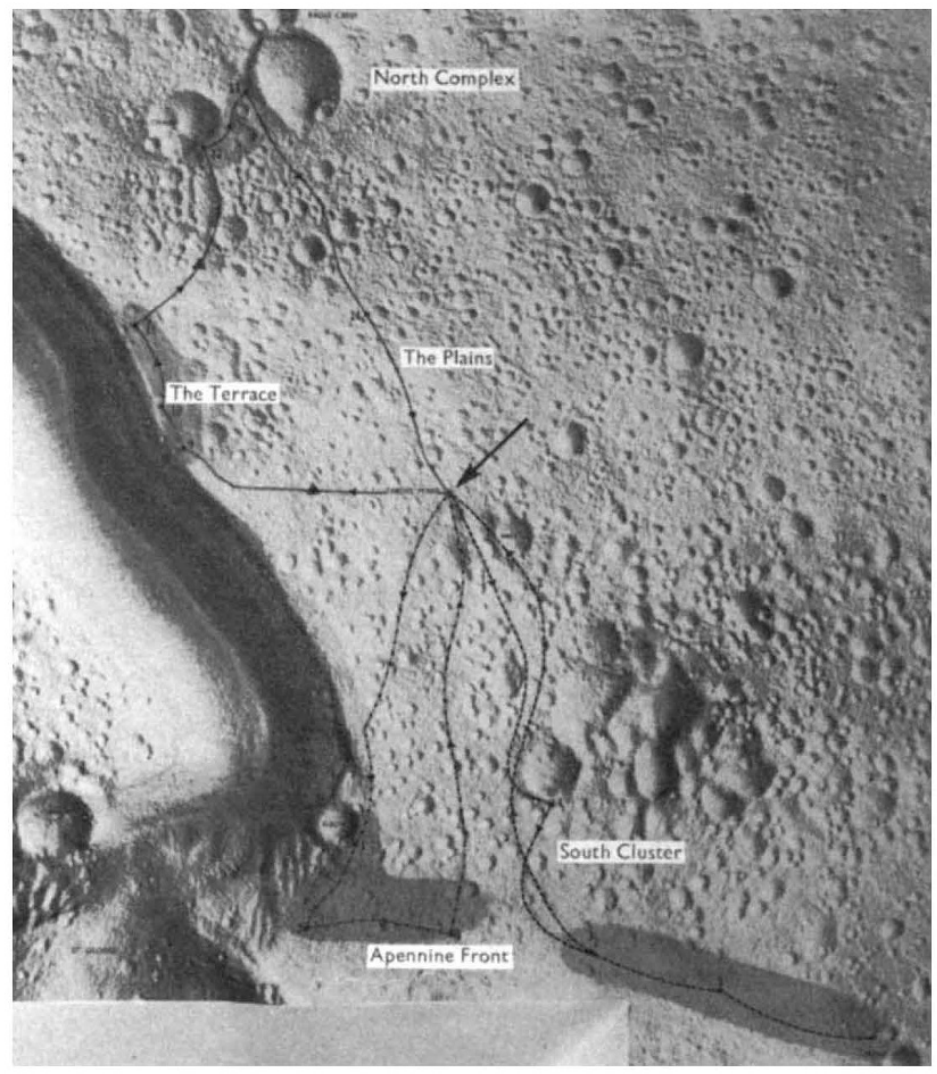

Relief map, prepared by NASA, of the region around the Apollo 15 landing site (indicated by arrow) several kilometres to the east of Hadley Rille. The map marks the projected routes for three excursions to sample material from the Apennine Mountain region, to study the Rille itself, to collect material from the mare-possibly one of the youngest on the Moon-and from the north complex which may be volcanic, and to investigate the cluster of craters south-east of the landing site.

IN spite of financially imposed restraints, it looks as if the tail of the Apollo space programme will bring in the scientific data which so far have been a secondary objective of the manned Moon missions. The recent report that the Fra Mauro rocks obtained from the Apollo 14 landing site are 500 million years younger than expected (see Nature, 232, 10 ; 1971) makes this an appropriate time to look forward to the next two missions and their scientific aims.

The next lunar flight projected is that of Apollo 15, which should be launched on July 26. Improvements to the Saturn launch vehicle have increased the payload which can be taken to the Moon, and NASA seems to have taken some note of the criticism that they are merely running a prestigious "taxi service". Innovations incorporated in Apollo 15 are aimed at extending the research opportunities available both in lunar orbit and on the Moon's surface, although the much vaunted Moon rover which the astronauts will drive on the Moon's surface could probably have been usefully replaced by more valuable scientific equipment.
Each of the next two Apollo craft will carry small ( 80 pound) satellites to be released into orbit around the Moon. The observations made by these satellites will include liaison with a large magnetometer which the Apollo 16 crew will set up on the lunar surface. If successful, this will fill one of the most important gaps left by the failure of the Apollo 13 mission. Comparisons of the flux at the lunar surface with satellite measurements of the solar wind can be expected to lead to further developments of the theory, put forward by C. P. Sonett and his colleagues, of the Moon's structure and its formation (see Nature, 230, 359 ; 1971).

By doubling the number of passive seismic stations on the Moon to a total of four, the Apollo 15 and 16 missions will provide the means for accurate seismic surveys of the Moon, and it can be expected that once three stations have been established the active seismic experiments left on the Moon will be used to discharge explosive charges. In particular, these experiments will help to determine the thickness of the lunar soil layer formed by meteoroid impact, and the depth of the upland basin fill in the old crater Descartes which is the target for Apollo 16.

Perhaps the most important of the other equipment to be left on the lunar surface are a far ultraviolet spectrometer, a cosmic ray detector and several solar wind experiments. Because the present economic climate makes it unlikely that any further experiments will be placed on the Moon by hand for a long time, this belated burst of scientific activity by NASA is welcome.

Apart from the scientific experiments, the Apollo 15 and 16 missions will be important for the straightforward study of the geography and geology of the Moon. The Apollo 15 landing site near the intriguing Hadley Rille is the first site to be visited which is situated far from the lunar equator, and, quite apart from the value of a close range inspection of the rille, the surface rocks and soil may show differences from those collected on earlier missions. Apollo 16 will, if all goes well, land in Descartes. This is another near equatorial landing site $\left(16^{\circ}\right.$ south and $9^{\circ}$ east of the centre of the near side of the Moon), and lies in the central lunar highlands at one of the highest regions of the Earth side hemisphere. Perhaps one of these two missions really will find the 4.5 thousand million year old rocks which do not seem to be present near Mare Imbrium.

\section{MEMBRANES}

\section{Liquid Paralfin}

from our Molecular Biology Correspondent PHYSICAL chemists who have taken ship on the treacherous waters of biology with the resolve not to get their feet wet have sooner or later to steer an uncomfortable course between the Scylla of physical intractability and the Charybdis of biological irrelevance. It is more commonly the latter that claims them. Once in a while, however, the promised land comes in view-a physically defined problem, directly amenable to the methods available, with a real biological message. A case in point is the structure and properties of lipid bilayers, which, there now seems no doubt, have a direct bearing on the interior of biological membranes. Paraffin structure, once an esoteric branch of physical chemistry, is now molecular biology. Perhaps the most elegantly conceived and successful approach has been that of McConnell who has used his spin-label technique to obtain information of remarkable refinement about the nature of the bilayer in time and space.

Much of the argument about bilayer structure has devolved on the degree of orientation of the paraffin chains. McFarland and McConnell (Proc. US 